\title{
Radiation studies on resistive bulk-micromegas chambers at the CERN Gamma Irradiation Facility
}

\author{
B. Alvarez Gonzalez ${ }^{a}$, J. Bortfeldt ${ }^{a}$, M.T. Camerlingo ${ }^{b}$ E. Farina* ${ }^{* a}$, P. lengo ${ }^{a}$, J. \\ Samarati $^{a}$, O. Sidiropoulou ${ }^{a c}$, J. Wotschack ${ }^{a}$ \\ ${ }^{a}$ CERN \\ ${ }^{b}$ Universita di Napoli Federico II e INFN \\ ${ }^{c}$ Bayerische Julius Max. Universitaet Wuerzburg \\ ${ }^{d}$ Universita di Pavia e INFN \\ E-mail: edoardo.maria.farinadcern.ch
}

\begin{abstract}
Two resistive bulk-micromegas chambers were installed in May 2015 at the CERN Gamma Irradiation Facility exposed to an intense gamma irradiation with the aim to study the detector behavior under high irradiation and the long-term aging. The chambers have an active area of $10 \times 10 \mathrm{~cm}^{2}$, strip pitch of $400 \mu \mathrm{m}$, an amplification gap of $128 \mu \mathrm{m}$ and a drift gap of $5 \mathrm{~mm}$. The desired accumulated charge of more than $0.2 \mathrm{C} / \mathrm{cm}^{2}$ has been reached for one of the chambers, equivalent to 10 years of HL-LHC operation. The efficiency, amplification, and resolution of the chamber after this long-term irradiation period will be compared with the performance of a non irradiated chamber.

In addition, the latest results of the measured particle rate as a function of the amplification voltage will be presented. These results will be compared with those obtained in October 2015 and presented in the last MPGD conference in 2015.
\end{abstract}

5th International Conference on Micro-Pattern Gas Detectors (MPGD2017)

22-26 May, 2017

Philadelphia, USA

\footnotetext{
* Speaker.
} 


\section{Introduction}

With the increase of the LHC luminosity expected in the next years, detector aging properties have become extremely relevant to ensure an adequate detector performance over many years of operation. A dedicated gamma irradiation facility (GIF++) [2] has been realized at CERN combining a $14 \mathrm{TBq}{ }^{137} \mathrm{Cs}$ source with a muon beam to test detectors in a high rate particle environment and accumulate the integrated charge expected at the LHC conditions in a reasonable time. The source can be shielded by filters, in order to tune the photon rate reaching the detectors.

Resistive Micromegas [1] has been chosen as detector technology for the ATLAS NSW upgrade [3]. Two $10 \times 10 \mathrm{~cm}^{2}$ detectors have been installed in the facility in May, 2015, with the goal of accumulating $0.2 \mathrm{C} / \mathrm{cm}^{2}$ and to evaluate the detector performance in a high rate environment. The two detectors, having a strip pitch of $400 \mu \mathrm{m}$, a drift gap of $5 \mathrm{~mm}$ and an amplification one of $128 \mu \mathrm{m}$, have been placed at a distance of about 1 metre from the source.

\section{Detector sensitivity}

The detector sensitivity to photons has been obtained by comparing the number of photons converting in the gas, reconstructed by means of the read-out electronics (observed particle rate) and the total number of photons crossing the gas gaps (simulated photon rate). APV-25 front-end ASICs and RD51 Scalable Readout System (SRS) [7] have been used for the data-taking. The photon rate has been evaluated in a Geant 4 simulation of the facility, where the precise geometry of the bunker and of the source has been used [6] and a realistic description of the detector has been implemented. In table 1 the sensitivity calculated for different attenuations of the photon flux, using the dedicated filters is reported. The $\gamma$ flux without any filter corresponds to a reconstructed converted particle rate of about $120 \mathrm{kHz} / \mathrm{cm}^{2}$.

\begin{tabular}{rrrr}
\hline Att. of $\gamma$ field & Obs. part. rate $\left(\mathrm{kHz} / \mathrm{cm}^{2}\right)$ & Sim. photon rate $\left(10^{7} \mathrm{kHz} / \mathrm{cm}^{2}\right)$ & Sensitivity \\
\hline 1 & 115000 & 7.70 & $\mathbf{0 . 0 0 1 4}$ \\
1.5 & 99130 & 5.21 & $\mathbf{0 . 0 0 1 9}$ \\
2.2 & 68052 & 3.72 & $\mathbf{0 . 0 0 1 8}$ \\
4.6 & 34590 & 1.89 & $\mathbf{0 . 0 0 1 8}$ \\
\hline
\end{tabular}

Table 1: The detector sensitivity, the simulated photon rate and the observed converted particle rate for different filter configurations.

\section{Detector performance under irradiation}

The rate capability of the detector has been tested by measuring the resolution of the detector to muons, while exposed to the photon flux. Details concerning the analysis can be found in Ref. [5]. Fig. 1 shows the resolution obtained as a function of the particle rate up to about $70 \mathrm{kHz} / \mathrm{cm}^{2}$. No degradation of the performance has been observed. On the secondary axis the most probable value (MPV) of the Landau distribution of the cluster charge of reconstructed muons has been plotted, showing no reduction of the detector amplification. 


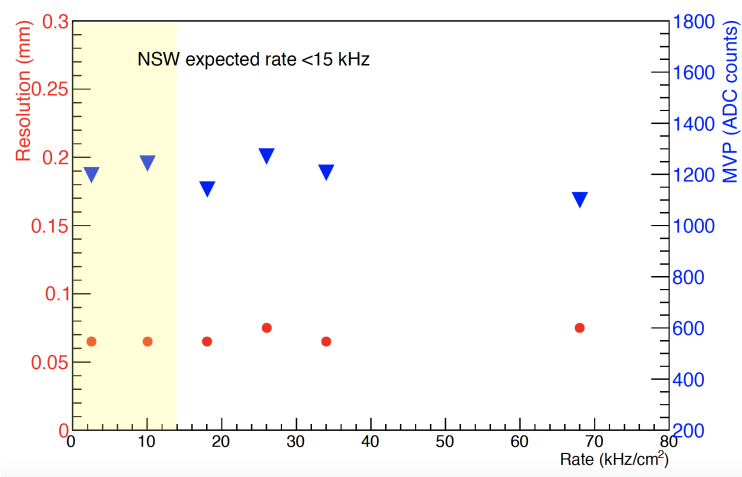

Figure 1: Detector resolution in a muon beam as a function of the photon rate up to $70 \mathrm{kHz} / \mathrm{cm}^{2}$. The blue triangles show the MPV value of the the muon cluster charge.

\section{Accumulated charge}

The expected accumulated charge over 10 years of HL-LHC operations is about $0.2 \mathrm{C} / \mathrm{cm}^{2}$. The two bulk chambers (called $\mathrm{T} 5$ and $\mathrm{T} 8$ in the following) have been exposed to photons for a total accumulated charge of about $0.3 \mathrm{C} / \mathrm{cm}^{2}$, as shown in Fig. 2. The different slopes are explained by changes in the position of the detectors or different attenuations of the source.

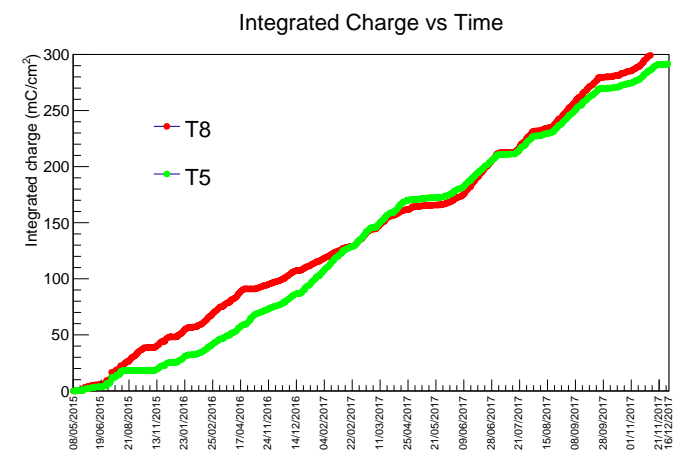

Figure 2: Observed accumulated charge as a function of time from May 2015 to December 2017. More than $0.3 \mathrm{C} / \mathrm{cm}^{2}$ have been accumulated, exceeding the goal of $0.2 \mathrm{C} / \mathrm{cm}^{2}$.

\section{Aging properties}

The aging of a gaseous detector can lead to a reduction of the amplification field, resulting in a reduction of the efficiency. Efficiency and gain measurements with ${ }^{55} \mathrm{Fe}$ have been performed comparing results obtained before and after accumulating more than $0.2 \mathrm{C} / \mathrm{cm}^{2}$. Very similar setup have been used in both cases, except for atmospheric conditions (pressure and temperature). Figure 3 shows the efficiency of both chambers to muons as a function of the amplification field. Despite of small differences at low voltage, attributable to atmospheric conditions, a plateau efficiency higher than $99 \%$ is reached. Figure 4 shows the gain as a function of the amplification voltage. Also in this case no sign of degradation is observed. 


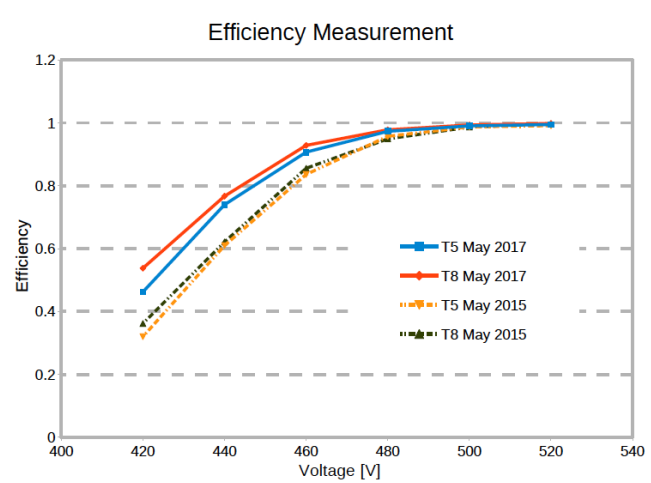

Figure 3: Efficiency to muons as a function of the amplification voltage for both Micromegas prototypes (T5 and T8) before and after the irradiation.

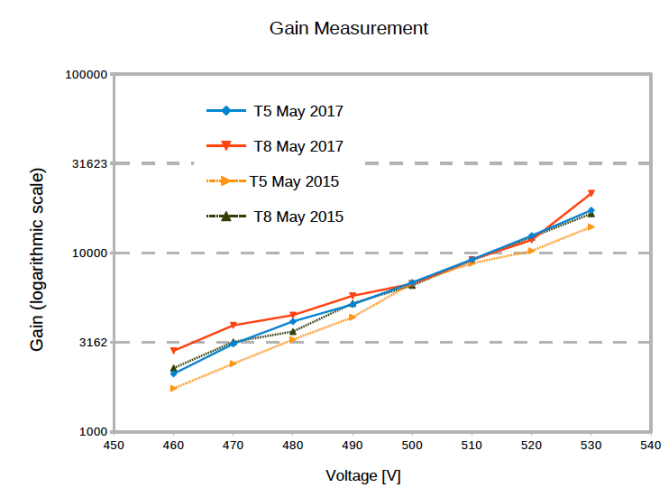

Figure 4: Amplification gain measured for both Micromegas prototypes (T5 and T8) before and after the irradiation. Also in this case no critical reduction of the gain is observed.

\section{Conclusions}

Two bulk resistive Micromegas prototypes have been exposed for more than two years to an intense photon flux at the GIF++ facility at CERN. Detector performance as a function of particle rate has been measured, observing no amplification or resolution reduction up to $70 \mathrm{kHz} / \mathrm{cm}^{2}$. The amplification and the efficiency have been measured before exposing the detectors to the photon irradiation and after accumulating $0.2 \mathrm{C} / \mathrm{cm}^{2}$. In conclusion no sign of aging has been observed, in agreement with previous results already published [4].

\section{References}

[1] T. Alexopoulos et al., Nucl. Instrum. Meth. A 640 (2011) 110.

[2] M. R. Jaekel et al., CERN GIF++, PoS (TIPP2014) 102

[3] ATLAS collaboration, ATLAS-TDR-20-2013.

[4] J. Galan et al., JINST 7, (2012) C01041

[5] 0. Sidiropoulou, et al., PoS ICHEP2016 (2016), C16-08-03

[6] D. Pfeiffer et al, https://arxiv.org/abs/1611.00299

[7] S. Martoiu et al., JINST 8 (2013) C03015 